\title{
Perfil higiênico-sanitário de um estabelecimento de gelados artesanais localizado em
}

\section{Juazeiro, BA}

\author{
Hygienic-sanitary profile of an artisanal ice cream establishment located in Juazeiro, BA \\ Perfil higiénico-sanitario de una heladería artesanal ubicada en Juazeiro, BA
}

Recebido: 06/12/2021 | Revisado: 11/12/2021 | Aceito: 18/12/2021 | Publicado: 20/12/2021

Renata Freire Alves Gondim

ORCID: https://orcid.org/0000-0003-4483-849X

Universidade de Pernambuco, Brasil

E-mail: renata.freire@upe.br

Jaqueline Damos da Silva

ORCID: https://orcid.org/0000-0003-3406-518X

Universidade de Pernambuco, Brasil

E-mail:jaqueline.damos@upe.br

Adriana da Silva Santos

ORCID: https://orcid.org/0000-0001-9539-7239

Universidade de Pernambuco, Brasil

E-mail:adriananutri.upe@gmail.com

Annayra Bruna de Sá Leal

ORCID: https://orcid.org/0000-0003-2991-5872

Universidade de Pernambuco, Brasil

E-mail:Annayra.leal@upe.br

Caroline Cabral dos Santos Silva

ORCID: https://orcid.org/0000-0003-4520-1554

Universidade de Pernambuco, Brasil

E-mail:Carolinecabral1296@gmail.com

Danielle Miranda Silva

ORCID: https://orcid.org/0000-0001-5787-3564

Universidade de Pernambuco, Brasil

E-mail:danielle.miranda@upe.br

Felipe Ithalo Ferreira da Silva

ORCID: https://orcid.org/0000-0002-1160-9546

Universidade de Pernambuco, Brasil E-mail:Felipe.ithalo@upe.br

Matheus Felipe Bernardino Nascimento

ORCID: https://orcid.org/0000-0003-3392-9652

Universidade de Pernambuco, Brasil

E-mail:matheus.bernardino@upe.br

Claudileide de Sá Silva

ORCID: https://orcid.org/0000-0002-3677-3000

Universidade de Pernambuco, Brasil

E-mail: claudileide.silva@upe.br

\begin{abstract}
Resumo
A indústria e a produção artesanal de gelados comestíveis é um segmento muito apreciado por grande parte da população, sendo bastante atrativo principalmente para crianças. Com isso, o objetivo desse artigo é avaliar as condições higiênico-sanitárias e o controle microbiológico em um empreendimento de gelados artesanais localizado em Juazeiro, Bahia. O presente artigo trata-se de um estudo descritivo transversal, qualitativo e quantitativo. As avaliações higiênicosanitárias foram realizadas através da aplicação do checklist conforme a RDC no 216/2004, ANVISA, e a análise microbiológica para a contagem de Staphylococcus aureus e de Coliformes totais, onde foram coletadas amostras de água utilizadas na produção de sacolés e das superfícies. Em relação às boas práticas de manipulação, essa micro empresa foi classificada como Grupo 2 (57\% de adequação). Já as análises microbiológicas apontaram a presença de S. aureus nos sacolés de maracujá ( 3 x $10 \mathrm{UFC} / \mathrm{g})$, porém apresentam-se toleráveis à legislação. $\mathrm{O}$ copo do liquidificador apresentou coliformes totais $\left(2,2 \times 10^{3} \mathrm{UFC} / \mathrm{cm}^{2}\right)$, onde essa contagem deve ser inferior à $2 \mathrm{UFC} / \mathrm{cm}^{2} \mathrm{em}$ superfícies. De acordo com a natureza das contaminações e o percentual de adequação encontrado, é notório a necessidade de adequação às boas práticas e um responsável técnico, como um nutricionista. Pois, diversos estudos já evidenciaram que quando da presença deste profissional, existe uma menor incidência de contaminações e melhor percentual de adequação.
\end{abstract}

Palavras-chave: Boas práticas; Coliformes totais; Gelados artesanais; Staphylococcus aureus. 


\begin{abstract}
The industry and the artisanal production of edible ice-cream products is a segment very appreciated by a large part of the population, being very attractive especially for children. This is a descriptive, cross-sectional, qualitative and quantitative study. The hygienic-sanitary evaluations were carried out by applying the checklist according to RDC No. 216/2004, ANVISA, and the microbiological analysis for the counting of Staphylococcus aureus and Total Coliforms, where water samples were collected from the bags, from the blender cup and a basin. Regarding good handling practices, this micro company was classified as Group 2 (57\% adequacy). The microbiological analysis indicated the presence of $\mathrm{S}$. aureus in passion fruit bags, but they are tolerable to the legislation. The blender cup showed total coliforms $(2.2 \mathrm{x}$ $10^{3} \mathrm{CFU} / \mathrm{cm}^{2}$ ), where this count should not exceed the threshold of $<100 \mathrm{CFU} / \mathrm{cm}^{2}$ on surfaces. The need for a professional such as a nutritionist is evident in order to meet all the necessary demands, from food preparation to transport, which would avoid food contamination.
\end{abstract}

Keywords: Good habits; Coliform totals; Handmade ice cream; Staphylococcus aureus.

\title{
Resumen
}

La industria y producción artesanal de helados comestibles es un segmento muy apreciado por gran parte de la población, siendo especialmente atractivo para los niños. Con ello, el objetivo de este artículo es evaluar las condiciones higiénico-sanitarias y el control microbiológico en una empresa de helados artesanales ubicada en Juazeiro, Bahía. Este artículo es un estudio descriptivo, transversal, cualitativo y cuantitativo. Las evaluaciones higiénico-sanitarias se realizaron aplicando el checklist según RDC No. 216/2004, ANVISA, y el análisis microbiológico para el conteo de Staphylococcus aureus y Coliformes totales, donde se utilizaron muestras de agua en la producción de bolsas y superficies. En cuanto a las buenas prácticas de manejo, esta microempresa fue clasificada como Grupo 2 (57\% de adecuación). Los análisis microbiológicos indicaron la presencia de S. aureus en bolsas de maracuyá (3 x $10 \mathrm{UFC} / \mathrm{g})$, pero son tolerables a la legislación. La copa de la licuadora mostró coliformes totales $\left(2.2 \times 10^{3} \mathrm{CFU} / \mathrm{cm}^{2}\right)$, donde este recuento debe ser menor a $2 \mathrm{CFU} / \mathrm{cm}^{2}$ en superficies. Según la naturaleza de las contaminaciones y el porcentaje de adecuación encontrado, es notoria la necesidad de adaptarse a las buenas prácticas y un técnico responsable, como un nutricionista. Porque, varios estudios han demostrado que cuando este profesional está presente, hay una menor incidencia de contaminación y un mejor porcentaje de adecuación.

Palabras clave: Buenas practicas; Totales de coliformes; Helado artesanal; Staphylococcus aureus.

\section{Introdução}

O consumo de alimentos artesanais têm aumentado significativamente nos últimos anos, e dentre eles os gelados. De acordo a Portaria $n^{\circ}$ 266, de 22 de setembro de 2005, do Agência Nacional de Vigilância Sanitária, os gelados comestíveis, como os picolés ou sacolés podem ser preparados a partir da emulsão de gorduras e proteínas, com ou sem a adição de outros ingredientes, conduzidos posteriormente ao congelamento, que tornam a sua consistência cremosa, refrescante e adocicada, amplamente atrativa e acessível à sociedade, especialmente ao público infantil (Paiva et al, 2016).

A perceptível transição na produção de gelados comestíveis de nível industrial a nível artesanal acompanha a expansão de suas versões alcoólicas e gourmet, viabilizam maior subsistência à população, através da redistribuição econômica e social, além da questão nutricional, que impulsionam a produção e a comercialização, propiciando a produção em aproximadamente 1.129 milhões de litros com consumo per capita previsto em 5,44 litros ao ano conforme as projeções relatadas pela Associação Brasileira das Indústrias e do Setor de Sorvetes no Brasil em 2017 (Veiga et al, 2020; Barcelos et al, 2019; Ferreira et al, 2020).

O elevado valor nutricional, o pH aproximadamente neutro, o armazenamento prolongado associado à elevada atividade de água (Aa) constituem influentes fatores intrínsecos à instalação e/ou proliferação microbiológica em decorrência da satisfatório composição (Hamú \& Cardoso, 2019; Andrade et al, 2017).

Além disso, os procedimentos higiênico-sanitários empregados ao decorrer da cadeia produtiva discriminada desde a recepção dos alimentos à preparação, armazenamento e comercialização são consideradas potenciais condicionantes ao desenvolvimento de Doenças Transmitidas por Alimentos (DTAs), principalmente quando associada a equipamentos e a estrutura operacional precária (Paiva et al, 2016; Ferreira et al, 2020). 
O possível tratamento térmico ineficiente e as secreções dos manipuladores atrelada à microbiota de gelados comestíveis podem promover a proliferação de Salmonella spp, Escherichia coli e Staphylococcus aureus pela integração de ovos e/ou laticínios à composição da preparação (Hamú \& Cardoso, 2019).

Desse modo, o objetivo do presente trabalho é avaliar as condições higiênico sanitárias e o controle microbiológico em um empreendimento de gelados artesanais localizado na cidade de Juazeiro, Bahia.

\section{Metodologia}

Trata-se de um estudo de caráter descritivo transversal, qualitativo e quantitativo, descrito por Pereira et al (2018), desenvolvido em agosto de 2021. A coleta de dados foi realizada em um empreendimento produtor de picolés artesanais localizado no centro do município de Juazeiro na Bahia, cidade localizada no sertão do São Francisco, Brasil.

\section{Instrumento de coleta}

O instrumento de coleta para determinação das condições higiênico-sanitárias do estabelecimento foi composto por uma lista de verificação de boas práticas de manipulação e fabricação de alimentos baseada nas RDC 216/2004, e CVS 5/2013, publicada pela Agência Nacional de Vigilância Sanitária (ANVISA) e Secretaria de Saúde do Estado de São Paulo, respectivamente. Os resultados obtidos através da referida lista foram classificados conforme seu percentual de adequação de acordo com a RDC n $n^{\circ}$ 275/2002, ANVISA, em que ao Grupo 1 pertencem os estabelecimentos que atingem de 76 a $100 \%$ de atendimento dos itens; no Grupo 2 de 51 a 75\%; e no Grupo 3 de 0 a $50 \%$ de atendimento dos itens. Para avaliação do risco de contaminação, também foi adotada a classificação de acordo com a RDC 267/2003, ANVISA, para o item qualidade da água.

Sendo assim, na lista de verificação foram abordados 177 itens distribuídos nas seguintes seções: I) Recebimento de mercadorias; II) Armazenamento; III) Equipamentos, móveis e utensílios; IV) Instalação e edificação; V) Higiene das instalações; VI) Manipulação dos alimentos; VII) Manipuladores de alimentos;VIII) Abastecimento de água; IX) Sanitários e vestiários; X) Resíduos sólidos-lixo; XI) Área de venda e XII) Controle de vetores.

Para calcular o percentual de conformidade, o número total de itens conformes pelo estabelecimento, foi dividido pelo total de itens aplicáveis ao mesmo, e então multiplicado por 100. O mesmo cálculo foi realizado para cada tópico que compôs toda a lista de verificação.

A análise microbiológica foi elaborada a partir de coletas de amostras da água utilizada na produção dos alimentos e higienização de utensílios, amostras de picolés artesanais de maracujá e de limão. Além disso, foram coletadas amostras de superfície de um copo de liquidificador e de recipientes utilizados tanto para a produção quanto armazenamento e transporte dos gelados.

As coletas das referidas amostras foram realizadas in loco, e as análises microbiológicas para a contagem de coliformes totais, Escherichia coli e Staphylococcus aureus utilizando o sistema de análise Petrifilm Coliform Count Plate e STX 3M®, respectivamente. Para classificação, foram consideradas adequadas as amostras que se encontravam abaixo do limite máximo conforme descrito na Instrução Normativa n ${ }^{\circ}$ 60, de 23 de dezembro de 2019 e Resolução de Diretoria Colegiada nº 12, de 2 de janeiro de 2001, da Agência Nacional Vigilância Sanitária. Para as amostras de água foram utilizadas placas Petrifilm Aqua $3 \mathrm{M} \circledast$, por ser um sistema simplificado de análises microbiológicas para a água, e classificadas em adequadas e inadequadas segundo a Portaria n ${ }^{\circ} 888 / 2021$, do Ministério da Saúde. Para amostras de superfície, foram consideradas satisfatórias, quando o número de colônias de coliformes totais não ultrapassou 2 por $\mathrm{cm}^{2}$ (Freitas et al, 2020). 


\section{Resultados e Discussão}

O estabelecimento analisado trata-se de um micro-empreendimento do ramo de gelados no qual o produto é confeccionado e comercializado, denominado como sacolé, os quais são picolés artesanais com ou sem a adição de laticínios.

\section{Análise das condições higiênico-sanitárias do estabelecimento}

Após a aplicação da lista de verificação, verificou-se que o estabelecimento apresentou adequação de $57 \%$, o classificando como Grupo 2. Resultado também preocupante foi descrito por Barbosa et al (2019), ao avaliar as condições higiênico sanitárias de duas sorveterias artesanais na cidade de Cuiabá/MT, constataram que ambas apresentaram alto risco de contaminação devido ao baixo percentual de adequação que apresentaram.

Na Tabela 1 são evidenciados os percentuais de adequação e não conformidade do total de itens analisados por seção que compôs a lista de verificação.

Tabela 1. Relação de grupo de itens avaliados através do check-list às boas práticas de manipulação de alimentos e seus respectivos percentuais de adequação, aplicados em um empreendimento produtor de gelados artesanais, na cidade de JuazeiroBA, 2021.

\begin{tabular}{|c|c|c|c|c|}
\hline Aspecto avaliado & Itens analisados & Conforme & Não conforme & Percentual de conformidade $(\%)$ \\
\hline I.Edificações e instalações & 28 & 19 & 9 & $67 \%$ \\
\hline II.Higienização & 13 & 10 & 3 & $76 \%$ \\
\hline $\begin{array}{l}\text { III.Controle integrado de vetores e } \\
\text { pragas }\end{array}$ & 3 & 3 & 0 & $100 \%$ \\
\hline IV.Abastecimento de água & 8 & 8 & 0 & $100 \%$ \\
\hline V.Manejo de resíduos & 7 & 6 & 1 & $85 \%$ \\
\hline VI.Manipuladores & 14 & 5 & 9 & $35 \%$ \\
\hline $\begin{array}{l}\text { VII.Matérias primas, ingredientes } \\
\text { e embalagens }\end{array}$ & 15 & 10 & 5 & $66 \%$ \\
\hline VIII.Preparação do alimento & 11 & 7 & 4 & $63 \%$ \\
\hline $\begin{array}{l}\text { IX.Armazenamento e transporte } \\
\text { dos alimentos preparados }\end{array}$ & 3 & 0 & 3 & $0 \%$ \\
\hline X.Exposição ao consumo & 8 & 4 & 4 & $50 \%$ \\
\hline XI.Documentação e registro & 14 & 0 & 14 & $0 \%$ \\
\hline XII.Responsabilidade & 2 & 0 & 2 & $0 \%$ \\
\hline
\end{tabular}

Fonte: Autores.

Os piores percentuais de adequação foram encontrados para as seções armazenamento e transporte dos alimentos preparados, documentação e registro, e a de responsabilidade, em que nenhum dos itens avaliados estavam adequados. Neste sentido, não existia documentação relacionada a implantação e controle das boas práticas de manipulação, como também a inexistência de responsável técnico.

Estes achados são preocupantes, pois no manejo do armazenamento e transporte de alimentos preparados, discriminados na categoria IX, nenhum dos itens analisados apresentou conformidade, constituindo, portanto, um dado alarmante tendo em 
vista as condições intrínsecas dos gelados artesanais, que tornam viável a sobrevivência e reprodução de patógenos, especialmente quando associada à condições externas favoráveis, como a inserção de sacolé a temperatura aquém do ideal (Vieira et al, 2020). Neste contexto, a importância deste controle é reforçada pelo descrito pela resolução SAA - 03, de 10 de janeiro de 2008, da Coordenadoria de Defesa Agropecuária de São Paulo, pois práticas irregulares frequentemente observadas em empreendimentos artesanais são a oscilação térmica durante o armazenamento e o manejo em condições higiênicas precárias (Paiva et al, 2016).

$\mathrm{O}$ item referente à documentação e registros analisados, avaliado no tópico XI, também obteve percentual de conformidade $0 \%$, onde encontram-se ausentes os documentos de boas práticas de manipulação e fabricação e procedimentos operacionais padronizados (POPs) e manual de boas práticas, corroborando ao comprometimento da qualidade e segurança dos alimentos. Este resultado foi semelhante ao relatado por Barbosa et al. (2019) ao avaliar quatro sorveterias na cidade de Cuiabá/MT, sendo duas delas artesanais, em que o percentual de três delas foi $0 \%$ para o item documentação e registros.

Segundo Verdum et al (2017), a inexistência desses documentos deduz a ausência de procedimentos padronizados e coordenados, portanto, é um parâmetro determinante que compromete a qualidade dos alimentos produzidos nos serviços de alimentação e fabricação. Ressalta-se que a adesão aos POPs e as boas práticas de manipulação e fabricação de alimentos são determinantes à garantia da saúde do consumidor, através do planejamento de condutas que proporcionem efetiva qualidade tanto nutricional quanto sanitária aos alimentos preparados, contudo, a sua efetiva aplicação requer a adequada capacitação dos manipuladores, para que possam conhecer a essencialidade dos procedimentos, seguindo assim, rigorosamente os protocolos prescritos (Santos et al, 2017).

Em relação à Responsabilidade técnica delimitada no item XII, pontuando um total de $0 \%$ de conformidade em decorrência da ausência de profissional capacitado para administrar o serviço em questão, considera-se que é o principal fator determinante à identificação da ausência de documentação e registros atrelada às inadequações quanto ao manuseio, armazenamento e transporte pelo manipulador de alimentos, consequente à falta de protocolos sistemáticos. Pois tais documentos são de competência do profissional habilitado o qual assume a responsabilidade técnica, em elaborar, implantar e manter os mesmos.

Esta situação pôde ser observada em um estudo observacional envolvendo 3 empreendimentos alimentícios localizados no campus da Universidade Federal do Pará, em que foi constatado, que dois restaurantes que não possuíam responsável técnico enquadraram-se na classificação do Grupo 2, circunscrito como regular, enquanto um restaurante, que contemplava assessoria de responsável técnico, sendo profissional nutricionista, pontuou 89,3\% de conformidade sendo enquadrado como bom no Grupo 1, conforme a RDC 275/2002 ${ }^{9}$, da Agência Nacional de Vigilância Sanitária. Tiboni (2017) também constatou melhoria significativa e representativa em sua avaliação higiênico-sanitária anterior e posterior à assessoria de responsável técnico com graduação em nutrição, corroborando ao segmento restrito dos protocolos planejamentos, visando a garantia à qualidade e segurança alimentar dos consumidores (Carvalho et al, 2016; Tiboni, 2017).

O segundo menor percentual de adequação foi verificado para a seção VI, discriminada em manipuladores, apresenta um total de conformidade 37\% em decorrência do emprego frequente de práticas higiênico-sanitária irregulares durante o processo de fabricação de sacolé, compreendendo desde a recepção dos ingredientes à preparação, acondicionamento em freezer e comercialização. Este resultado também pode estar atrelado à ausência do responsável técnico, uma vez que é de sua competência a capacitação e monitoramento do seguimento às boas práticas por parte dos manipuladores manipuladores de alimentos.

É importante ressaltar que na maioria dos casos de surtos de origem alimentar, considera-se como os principais difusores: as irregularidades praticadas na cadeia produtiva provenientes da distração visual e sonora, desleixo e escasso 
treinamento quanto às boas práticas de manipulação em responsáveis pela manipulação de alimentos, sendo estes frequentemente interligados às contaminações microbiológicas (Santos et al, 2021; Souza et al, 2021).

Resultados intermediários foram verificados para as seções X. Exposição ao consumo, VIII. Preparação do alimento, VII. Matérias primas, ingredientes e embalagens, Edificações e instalações, que variaram de 50 a $67 \%$ de adequação, enquanto que os melhores resultados foram para Higienização, Manejo de resíduos, Controle integrado de vetores e pragas e abastecimento de água, que variou de 76 a 100\% de adequação sendo este percentual atingido pelas duas últimas seções citadas.

Para o abastecimento de água, a conformidade foi de $100 \%$ e assim, foi considerado de baixo risco para contaminação por água, de acordo com a RDC 267/2003, ANVISA. Estes dados corroboram com os descritos por Silva et al (2016) e Miranda et al (2019), ao avaliarem as condições higiênico sanitárias de sorveterias em algumas cidades brasileiras, em que apesar da baixa adequação em outros itens, a potabilidade da água foi assegurada. Este é um dado considerado importante, pois a potabilidade da água é crítica para a produção de sorvetes e picolés já que pode comprometer qualidade ou segurança tanto dos próprios produtos como dos processos (Silva et al., 2016).

\section{Análise microbiológica}

$\mathrm{Na}$ análise do risco de transmissão de microrganismos para alimentos e consequentemente para os consumidores, foi possível observar que ele possivelmente irá aumentar quando os controles sobre as práticas de higiene no manuseio dos alimentos são insuficientes, o que pode ser visto no estudo (Tabela 1).

Nesse contexto, os surtos de DTAs podem ser difundidos por bactérias da família enterobacteriaceae, como a Salmonella spp e Escherichia coli, que são consideradas microrganismos de maior repercussão à assistência hospitalar em decorrência do potencial risco ao desenvolvimento de severos quadros de toxinfecção alimentar, podendo encaminhar o indivíduo à obtido, principalmente aqueles condicionados à imunossupressão e/ou debilitados (Andrade et al, 2017).

Conforme pode-se observar na análise dos resultados apontados pela lista de verificação, estes se refletiram nos resultados das análises microbiológicas dos alimentos (Tabela 2). Sendo o resultado da água concordante com o apontado através da aplicação da lista de verificação, em que a adequação em 100\% refletiu na não contaminação da água.

Tabela 2. Contagem de microrganismos presentes em utensílios, alimentos e água de um empreendimento de gelado artesanal localizado em Juazeiro/ BA, Nordeste, Brasil.

\begin{tabular}{lccc}
\hline ITENS & STAPHYLOCOCCUS AUREUS & E. COLI & COLIFORMES TOTAIS \\
\hline Água & 0 & 0 & 0 \\
Sacolé de maracujá & $3 \times 10 \mathrm{UFC} / \mathrm{g}$ & 0 & $2,9 \times 10^{2} \mathrm{UFC} / \mathrm{g}$ \\
Sacolé de limão & 0 & 0 & $1,4 \times 10^{2} \mathrm{UFC} / \mathrm{g}$ \\
Copo de liquidificador & - & - & $2,2 \times 10^{3}{\mathrm{UFC} / \mathrm{cm}^{2}}^{2}$ \\
Bacia & - & - & $<1,0{\mathrm{UFC} / \mathrm{cm}^{2}}^{2}$ \\
\hline
\end{tabular}

Fonte: Autores.

Ao realizar análise microbiológica das amostras de picolés artesanais, no de sabor maracujá, verificou-se contagem de Staphylococcus aureus equivalente à 3,0 x $10 \mathrm{UFC/g}$, apesar de adequado conforme parâmetro estabelecido pela RDC $\mathrm{n}^{\circ}$ 12/2001, da Agência Nacional de Vigilância Sanitária, que limita S. aureus à no máximo 5 x $10^{2}$ UFC/g em gelados comestíveis 
e produtos especiais gelados a base de leite e produtos lácteos, assim como se encontra adequado conforme limites toleráveis a IN n60/2019, ANVISA, respeitando o limiar tolerável circunscrito em $10^{4} \mathrm{UFC} / \mathrm{g}$.

Estudos trazem que por meio das mãos, os manipuladores podem desempenhar papel importante na disseminação desses microrganismos, principalmente por meio dos alimentos por eles manuseados (Maia, 2017; Santos et al, 2021). Sendo assim, os teores obtidos na amostra de sacolé de maracujá podem apontar para bactérias presentes no próprio manipulador e que durante o preparo e manipulação pode ter transferido esses microrganismos para o alimento (Oliveira et al, 2019). Ressalta-se que os portadores nasais de S. aureus ao manipularem alimentos podem se tornar importantes fontes de contaminação para os alimentos (Almeida \& Morales, 2021).

Em ambas amostras de picolés analisadas foi encontrada a presença de coliformes totais, assim como no copo do liquidificador. Frequentemente as mãos são meios propícios à transferência de microrganismos para os alimentos, assim como utensílios mal higienizados podem se constituir fontes de transmissão de microrganismos para os alimentos. Nas mãos, os microrganismos mais encontrados são os Enterobacteriaceae e Staphylococcus aureus (Yamaguchi et al, 2021).

No copo do liquidificador foram detectadas coliformes totais estimados em $2,2 \times 10^{3} \mathrm{UFC} / \mathrm{cm}^{2}$, portanto, sendo considerada além do parâmetro delimitado por Freitas, o que pode apontar para uma higienização inadequada da infra-estrutura, equipamentos e utensílios (Santos et al, 2021).

A presença de coliformes totais em alimentos processados, segundo Oliveira et al (2019), é considerada uma indicação útil de contaminação pós-sanitização ou pós-processo, evidenciando assim, tanto as práticas de higiene e sanitização quanto a frequência aquém dos padrões requeridos para o processamento de alimentos (Maia, 2017).

A contaminação pode ocorrer durante o processo de higienização (assepsia) e preparação do alimento. Logo, sem a devida desinfecção, pode acontecer contaminação cruzada ${ }^{27}$. E desse modo, apontando este fatores como possível justificativa para a contaminação encontrada, uma vez que a água utilizada no procedimento não apresentou contaminação, atendendo dessa forma, aos parâmetros circunscritos pela Portaria no 888/2021, do Ministério da Saúde, onde prevê a inocuidade como prérequisito à regular distribuição hídrica quanto à análise microbiológica (Neto et al, 2018).

Estes resultados reforçam a importância de práticas adequadas de higienização e manipulação dos alimentos, e refletem os dados apontados através da aplicação da lista de verificação. Diante do diagnóstico foi possível constatar que a ausência do responsável técnico pode estar relacionada aos resultados encontrados, os quais são passíveis de prevenção e controle por profissional devidamente habilitado.

\section{Conclusão}

O presente trabalho constatou índices irregulares no controle higiênico-sanitário da empresa sob estudo, as quais poderiam ser evitadas pela ação e controle de um responsável habilitado.

Os resultados apresentados demonstram a necessidade urgente de contratação de um Responsável técnico a fim de investir na capacitação dos manipuladores, no armazenamento e também no transporte dos alimentos através da aplicação do Manual de Boas Práticas de Fabricação e Procedimentos Operacionais Padrão (POPs), corroborando para menores índices de contaminação e possível propagação das doenças transmitidas por alimentos.

Neste sentido, torna-se notório a importância da atuação do nutricionista no setor da produção a fim de conduzir e adequar as etapas no preparo, manipulação, armazenamento e transporte, a qual possivelmente evitaria a contaminação dos alimentos, preservando a saúde dos consumidores e dos próprios manipuladores de alimentos. 


\section{Referências}

Almeida, P. C., \& Morales, B. F. (2021). Análise das condições microbiológicas e higiênico-sanitárias da comercialização de pescado em mercados públicos de Itacoatiara, Amazonas, Brasil. Brazilian Journal of Development, 7(3), 32247-32269.

Andrade, A. P., Luche, D. E. D., Cervelatti, E. P., \& Matos, D. J. (2017). Aspecto higiênico-sanitário de sorvetes artesanais e industriais: análise da variabilidade genética e da resistência a antimicrobianos em Escherichia coli isoladas. Demetra, 12(2), 493-508.

Agência Nacional de Vigilância Sanitária (2019). Instrução Normativa N ${ }^{\circ}$ 60, de 23 de Dezembro De 2019 - Dispõe sobre os padrões microbiológicos para alimentos. Diário Oficial da União, Brasil. https://www.in.gov.br/en/web/dou/-/instrucao-normativa-n-60-de-23-de-dezembro-de-2019-235332356.

Agência Nacional de Vigilância Sanitária. (2002). Resolução no 275, de 21 de outubro de 2002. Dispõe sobre os Procedimentos Operacionais Padronizados e a lista de verificação das boas práticas de fabricação em estabelecimentos produtores/industrializadores de alimentos. Diário Oficial da República Federativa do Brasil, Brasília, DF. http://bvsms.saude.gov.br/bvs/saudelegis/anvisa/2002/anexos/anexo_res0275_21_10_2002_rep.pdf.

Agência Nacional de Vigilância Sanitária. (2004). Resolução no 216 de 15 de setembro de 2004. Dispõe sobre as boas práticas para serviço de alimentação. Diário Oficial da República Federativa do Brasil. https://bvsms.saude.gov.br/bvs/saudelegis/anvisa/2004/res0216_15_09_2004.html.

Barcelos, S. C., Silva, E. F., Batista, E. M., Santos, S., Sá, D. M. A. T., \& Monte, A. L. S. (2019). Qualidade microbiológica e física - química de sorvetes sabor chocolate comercializados na cidade de Limoeiro do Norte, Ceará, Brasil. Segur. Aliment. Nutr., 26,1 - 14.

Carvalho, L. S. C., Ribeiro, M. S. S., Souza, C. L., Nascimento, V. H. A. (2016). Boas práticas e qualidade sanitária dos alimentos servidos em restaurantes do tipo self-service no Campus da Universidade Federal do Pará. Segurança Alimentar e Nutricional. Pará, 23(2):924-932.

Centro De Vigilância Sanitária (2013). Portaria CVS 5, de 09 de abril de 2013. Aprova o regulamento técnico sobre boas práticas para estabelecimentos comerciais de alimentos e para serviços de alimentação, e o roteiro de inspeção, anexo. Secretaria De Estado Da Saúde, SÃO PAULO [s.l.]: , [s.d.]. http://www.cvs.saude.sp.gov.br/up/PORTARIA\%20CVS-5_090413.pdf.

Defesa Agropecuária. Resolução SAA - 03, de 10 de janeiro de 2008. Dispõe normas técnicas sobre as condições higiênico-sanitárias mínimas necessárias para aprovação, funcionamento e reaparelhamento dos estabelecimentos destinados a leite e produtos lácteos. Brasil, São Paulo. https://www.defesa.agricultura.sp.gov.br/legislacoes/resolucao-saa-03-de-10-01-2008,812.html.

Ferreira, N. M. S., \& Boênio, J. A. (2020). Avaliação Física, Química e Microbiológica de “Geladinho" de Tamarindo Comercializado em Morrinhos-GO. Hig. Alimentar 2020; 34(291):1-8.

Ferreira, N. M. S., \& Boênio, J. A. Avaliação Física, Química e Microbiológica de "Geladinho" de Tamarindo Comercializado em Morrinhos-GO. Hig. Alimentar, 34(291), 1-8.

Freitas, T. P., Costa, T. D., Ximenes, G. R., \& Silva, C. S. (2020). Condições higiênico-sanitárias de padarias de uma cidade do sertão pernambucano. Nutrição em Foco: uma abordagem Holística, 2, 548-563.

Fonseca, L. C., Azevedo, G. H. M., Santana, R. M., \& Baptista, A. B. (2019). Diversidade bacteriana em superfícies de restaurantes de Palmas-TO. Revista de Patologia do Tocantins, 6(2), 10-14.

Hamú, J. R. P. N., \& Cardoso, A. M. (2019). Avaliação microbiológica de sorvetes comercializados em Goiânia-GO. Revista RBAC, 50(4), 351-357.

Maia, M. O. (2017). Avaliação das condições higiênico-sanitárias de uma lanchonete no município de Limoeiro do Norte-CE. RevInter, 10(1), 45-56.

Ministério da Saúde. Portaria $n^{\circ} 888$, de 4 de maio de 2021. Dispõe sobre os procedimentos de controle e de vigilância da qualidade da água para consumo humano e seu padrão de potabilidade. Brasil. https://www.in.gov.br/en/web/dou/-/portaria-gm/ms-n-888-de-4-de-maio-de-2021-318461562.

Neto, A. F. N, Vasconcelos, C. E. C., \& Figueiredo, E. L. (2018). Avaliação higiênico-sanitária e microbiológica de estabelecimentos que comercializam açaí (Euterpe oleracea Mart.) em Igarapé-Miri - Pará. Brazilian Journal of Food Research, 9(2), 15-29.

Oliveira, A. G. M., Melo, L., Gomes, D. B. C., Peixoto, R. S., Leite, D. C. A., Leite, S. G. F., Colares, L. G. T., \& Miguel, M. A. L. (2019). Condições higiênicosanitárias e perfil da comunidade microbiana de utensílios e mesas higienizadas de um serviço de alimentação localizado no Rio de Janeiro. Braz. J. Food Technol, 22,1-14.

Paiva, Y. F., Silva, E. V., Araújo, A. S., Azevedo, P. T. M., \& Souza, T. C. A. (2016). Condições higiênico-sanitárias de sorvetes do tipo italiano (soft), comercializados em Pombal, Paraíba. Revista Verde de Agroecologia e Desenvolvimento Sustentável. Pombal, 11(3), 75-79.

Pereira, A. S., Shitsuka D. M., Parreira, F. J., \& Shitsuka, R. (2018). Metodologia da pesquisa científica. UFSM. 1, 1-119.

Santos, A. A., Benício, V. C., Silva, J. S., Silva, J. B., Lima, A. C. S. A., Albuquerque, J. M., Filipe, A. C. J., Silva, M. V. F., \& Silva C. S. (2021). Avaliação do gerenciamento de desperdícios e das condições higiênico-sanitárias de pizzaria localizada no norte da Bahia. Research, Society and Development. Bahia, 10 (6), 1-13.

Souza, A. O., Passos, E. V. B., Gonçalves, M. I. A., Coelho, R. L. L., \& Silva, C. S. (2021). Perfil higiênico-sanitário de um restaurante comercial no interior baiano. Research, Society and Development, Bahia, 10(2), 1-8.

Soragni, L., Barnabe, A. S., \& Mello, T. B. C. (2019). Doenças transmitidas por alimentos e participação da manipulação inadequada para sua ocorrência: uma revisão. Estação Científica (UNIFAP), 9(2), 19-31.

Santos, G. M., Costa, I. R., Oliveira, J. M. S., Saldanha, N. M. V. P., Souza, P. V. L., \& Carvalho, A. C. S. (2017). Avaliação das Condições Higiênico-Sanitárias e Físico-Estruturais de Panificadoras de uma Cidade do Nordeste Brasileiro. Rev. UNINGÁ Review, 32(1), $159-169$.

Silva, H. L. A.; Vieira, A. H.; Balthazar, C. F.; \& Cruz, A. G. (2016). Avaliação das boas práticas de fabricação em uma indústria de sorvetes de Niterói-RJ, Brasil. In: XXV Congresso Brasileiro de Ciência e Tecnologia de Alimentos, Gramado-RS. Gramado: FAURGS, p. 1-5. 
Research, Society and Development, v. 10, n. 17, e01101724439, 2021

(CC BY 4.0) | ISSN 2525-3409 | DOI: http://dx.doi.org/10.33448/rsd-v10i17.24439

Tiboni, G. S. (2017). Avaliação das condições higiênico-sanitárias antes e após assessoria do nutricionista em um restaurante comercial do município de Cotia, SP. Revista Higiene Alimentar, 31(274/275), 60-65.

Vieira, J. N., Silva, R. M., Santos, L. S., Pereira, Y. L., Garcia, L. G. C., \& Santos, P. A. (2020). Estudo de propriedades físicas de sorvete soft serve durante a estocagem. Research, Society and Development, 9(10), 1-12.

Veiga, S. M. O. M., Antonacio, N. R., \& Belmonte, M. G. (2020). Qualidade microbiológica de alimentos oriundos do comércio ambulante e intervenção educativa. Brazilian Journal of Development. Curitiba, 6(3), 14979-14997.

Yamaguchi, K. K. L., Costa, A. B. P. L., Oliveira, A. L. F. M., Costa, B. K. B. S., \& Yamaguchi, H. K. L. (2021). Conhecer para prevenir: boas práticas de higienização e manipulação de açaí. Revista Eletrônica de Extensão - Extensio; 18(38):44-59. 